\title{
A multi-item Physician Global Assessment scale to assess psoriasis disease severity: validation based on four phase III tofacitinib studies
}

\author{
Kristina Callis Duffin ${ }^{1}$, Andrew G. Bushmakin ${ }^{2}$, Joseph C. Cappelleri ${ }^{2}$, Lotus Mallbris ${ }^{3}$ and Carla Mamolo ${ }^{2^{*}}$ (1)
}

\begin{abstract}
Background: Several different Physician Global Assessment (PGA) versions have been used in clinical studies as a co-primary end point to evaluate psoriasis severity. Tofacitinib is an oral Janus kinase inhibitor. We performed an analysis of the PGA using data from studies of tofacitinib in moderate to severe chronic plaque psoriasis.

Methods: Data from 3641 patients with moderate to severe chronic plaque psoriasis, enrolled in one of four phase III tofacitinib studies (OPT Pivotal 1 and 2, OPT Compare and OPT Retreatment), were used to evaluate a three-item PGA scale.

Results: Confirmatory Factor Analyses showed that equal weighting of the three items (erythema, induration and scaling) was appropriate. The PGA demonstrated acceptable test-retest reliability (Intraclass Correlation Coefficient, 0.7) and internal consistency (Cronbach's Coefficient Alpha $\geq 0.9$ at primary time points). The Clinically Important Difference was estimated as 0.55 (95\% confidence interval: 0.546-0.563). Known-group validity was shown by demonstrating that PGA scores could discriminate between different degrees of disease severity. The PGA was significantly correlated with other clinical end points in the studies (Psoriasis Area and Severity Index, $r=0.75-0.79$; Dermatology Life Quality Index, $r=0.44-0.57$; Patient Global Assessment, $r=0.66-0.72$ ).

Conclusions: Consistent with previous findings from a phase II study, these results indicate that this PGA is a valid, reliable instrument for evaluating disease severity in clinical studies of psoriasis.
\end{abstract}

Keywords: Psoriasis, Physician Global Assessment, Tofacitinib

\section{Background}

The Physician Global Assessment (PGA) is a frequently used co-primary end point in psoriasis clinical trials. The PGA was introduced in 1998 by a US Food and Drug Administration panel as the preferred tool to assess and record the severity of disease in clinical studies, and typically rates a patient's disease from 'clear' to 'severe' or 'very severe' $[1,2]$. The PGA provides a simple subjective measurement of the clinical signs of psoriasis, typically erythema, induration and scaling, across the whole body [3]; however, there is currently no recognised standard definition of the PGA. Multiple

\footnotetext{
* Correspondence: carla.m.mamolo@pfizer.com

${ }^{2}$ Pfizer Inc, 445 Eastern Point Road, Groton, CT 06340, USA

Full list of author information is available at the end of the article
}

versions are currently in use, all varying in terms of the number and description of the items (or symptoms) assessed, and the point values used to rate each item, with the most common PGA versions using five- to sixpoint scales [3].

Tofacitinib is an oral Janus kinase inhibitor. A threeitem version of the PGA was developed for use in psoriasis clinical trials of tofacitinib [4]. This PGA scale asks physicians to rate erythema, induration and scaling, individually, on a five-point scale (from $0=$ no symptom to $4=$ severe). The total score is the mean of the three item scores, each having an equal weighting [4]. PGA scores based on a multi-item scale are less likely to be affected by random variations than scores based on single-item scales [5], and previous findings from a

(C) The Author(s). 2019 Open Access This article is distributed under the terms of the Creative Commons Attribution 4.0 International License (http://creativecommons.org/licenses/by/4.0/), which permits unrestricted use, distribution, and 
phase II clinical trial demonstrated the reliability and validity of the three-item PGA for the assessment of psoriasis severity [4].

The objective of the present study was to perform a more intensive and rigorous analysis of the PGA using data from four phase III studies of tofacitinib in moderate to severe chronic plaque psoriasis. This comprised validation of the PGA scoring algorithm using Confirmatory Factor Analysis (CFA), assessment of reliability and internal consistency of PGA measurements; definition of the Clinically Important Difference (CID); evaluation of the ability of the PGA to discriminate between different degrees of disease severity; and correlation of the PGA with other clinical outcome measures.

\section{Methods}

\section{Studies and patients}

Data from four phase III clinical studies of tofacitinib in patients with psoriasis (OPT Pivotal 1 [6], OPT Pivotal 2 [6], OPT Compare [7] and OPT Retreatment [8]) were used. Full details of the studies have been described elsewhere [6-8]. Briefly, OPT Pivotal 1 and 2 (ClinicalTrials.gov identifiers: NCT01276639 and NCT01309737) were similar 52-week phase III studies investigating tofacitinib $5 \mathrm{mg}$ twice daily (BID) and $10 \mathrm{mg}$ BID versus placebo [6].

The primary assessment time point was week 16 . OPT Compare (NCT01241591) compared tofacitinib $5 \mathrm{mg}$ BID or $10 \mathrm{mg}$ BID with etanercept $50 \mathrm{mg}$ twice weekly and placebo; the primary end point was assessed at week 12 [7]. OPT Retreatment (NCT01186744) was a treatwithdrawal-retreatment study of tofacitinib $5 \mathrm{mg}$ BID and $10 \mathrm{mg}$ BID versus placebo for up to 56 weeks [8].

Patients in all four studies were $\geq 18$ years of age, diagnosed with chronic ( $\geq 12$ months) plaque psoriasis, had a Psoriasis Area and Severity Index (PASI) score of $\geq 12$, a PGA of moderate or severe, and psoriasis that involved at least $10 \%$ of their body surface area [6-8]. All patients were candidates for systemic or phototherapy [6-8]; in OPT Compare, patients had to have failed to respond to, had a contraindication to, or been intolerant to, at least one conventional systemic therapy (including ultraviolet therapy) approved for plaque psoriasis treatment [7]. For this analysis, data from the primary efficacy assessment at week 24 (continuous treatment) were used [8].

\section{Assessments}

The co-primary end points in all four studies were the PGA and PASI. The PGA assessed three items (erythema, induration and scaling) on a scale from $0=$ clear to $4=$ severe. Items were rated separately across all psoriatic lesions and scored from 0 to 4 based on morphological descriptors. Severity rating scores for each item were summed and the mean taken; the mean was rounded to the nearest integer to determine the PGA score.

The PASI is a summary score evaluating the three signs of lesion severity (erythema, induration and scaling) according to a five-category scale for each of four anatomical regions of the body (head/neck, upper extremities, trunk and lower extremities) [1]. Higher PASI scores represent increasing psoriasis severity.

Where justified, evaluations were conducted using pooled data from all four studies to streamline and generalise results, as well as interpretation.

\section{Confirmatory Factor Analysis}

CFA was used to test the fit of the PGA measurement model. Bentler's Comparative Fit Index (CFI) was used as a measure of fit of the model with the data. Acceptable fit was defined as a CFI >0.9; in addition, path coefficients had to be statistically significant and standardised path coefficients had to be $>0.4$ [9-11]. The analysis was performed both with path coefficients constrained to be equal (reflecting the PGA scoring algorithm having equal weighting for each item) and with such constraints removed (Fig. 1).

\section{Test-retest reliability}

The PGA was performed for each patient on at least two different occasions under a relatively stable set of conditions before treatment intervention, allowing assessment of instrument stability and replicability. Test-retest reliability was evaluated by estimating a Dermatology Life Quality Index (DLQI) based on pooled PGA data. All available pre-treatment data (screening and baseline) were used. In addition, sensitivity analyses were performed using data from each study separately. An Intraclass Correlation Coefficient $\geq 0.70$ was considered satisfactory $[12,13]$.

\section{Internal consistency reliability}

Internal consistency reliability was assessed by calculating Cronbach's Coefficient Alpha and corrected item-tototal correlations (correlation of an item with the total score excluding that item) at baseline and the primary assessment time points. A Cronbach's Coefficient Alpha of $\geq 0.7$ was considered acceptable $[12,13]$.

\section{Clinically Important Difference}

CID for the PGA was defined by using a repeated measures model [13] to estimate the relationship between PGA and Patient Global Assessment (PtGA) scores. A linear relationship was imposed where PGA was the outcome and PtGA was a continuous anchor. This model incorporated all available data across all time points for all studies from baseline to the primary assessment time point. The mean difference on the PGA for a one- 


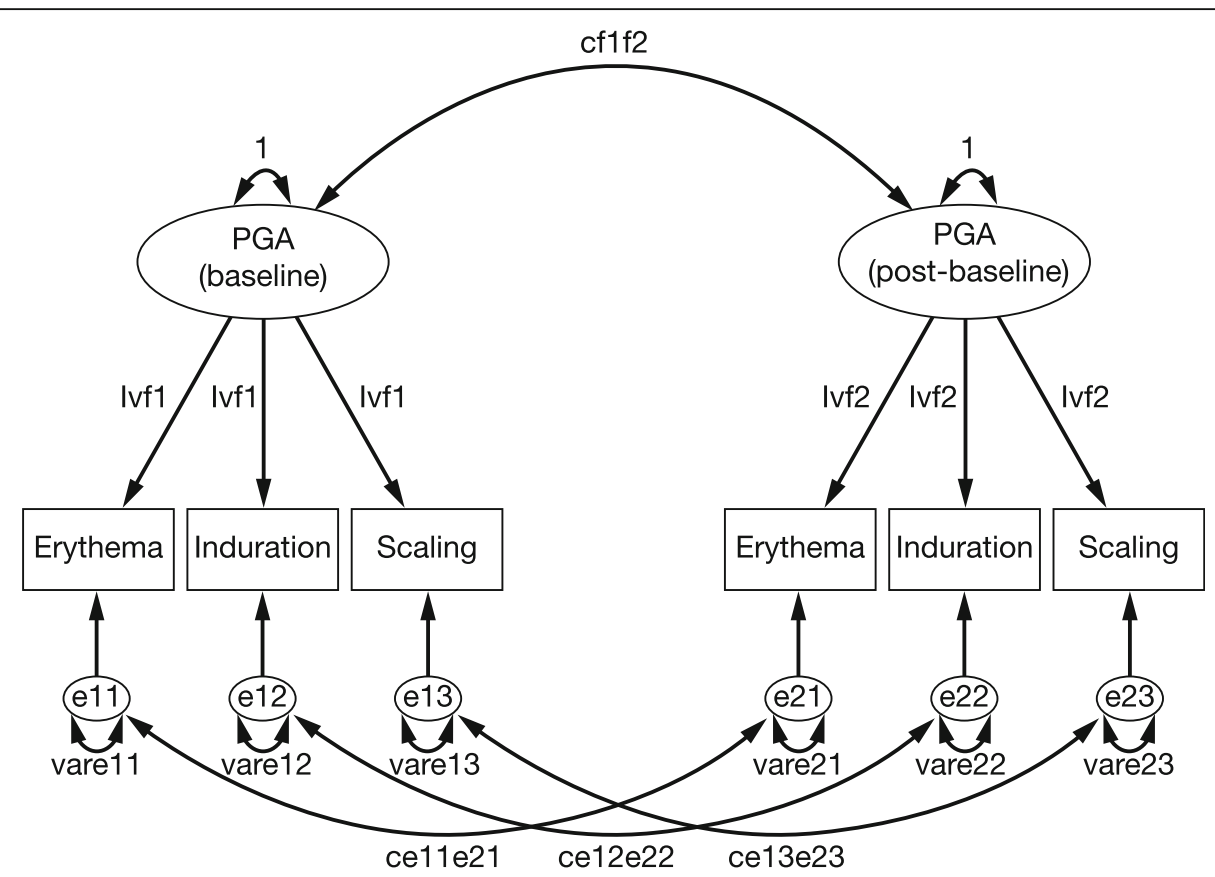

Fig. 1 Pathways in the measurement model used in the Confirmatory Factor Analysis in phase III studies. Equal weighting of each item (erythema, induration, scaling) is assumed. Ovals represent unobserved (latent) factors (i.e., PGA at baseline and post-baseline); rectangles represent observed items (e.g., erythema, induration, scaling). Loadings from PGA to each item are symbolised by 'Ivf1' (baseline) and 'Ivf2' (postbaseline). Residual or error terms represent all factors influencing variability in an item other than the latent factor that precedes and predicts that item. Error terms begin with 'e' and end with two digits indicating the item measured (e.g., 'e11' = error term for erythema and corresponding variances begin with 'var'). Covariances between pairs of error terms (latent factors) begin with ' $c$ ' (e.g., 'ce11e21' = covariance of the error term between erythema at baseline and at post-baseline). PGA Physician Global Assessment

category difference on the PtGA was taken as the estimated CID.

A sensitivity analysis was performed with PtGA as a categorical anchor. Doing so does not impose any functional relationship between an outcome and an anchor.

\section{Known-group validity}

For known-group validity [13], a repeated measures model was applied to assess the relationship between PGA and PASI. Pooled data for all four studies were analysed. PGA score was used as the outcome and PASI score as a categorical anchor (and hence no functional relationship was imposed). This model included all available data at all time points for all studies from baseline to the primary assessment time point. Seven categories were created: category 0 (observations when patient PASI score was exactly 0, i.e., 'healthy') to category 6 (observations when patient PASI score was in the range of 40 to 72), indicative of severe disease.

\section{Convergent and divergent validity}

Convergent and divergent validity [13] were assessed by determining the correlation of the PGA with the PtGA, PASI and DLQI. Evidence for convergent validity was based a priori on a Pearson correlation coefficient of $\geq$
0.40, consistent with a meaningful correlation [14]. Regarding divergent validity, evidence was based on a Pearson correlation coefficient of $\leq 0.30$, consistent with a less than medium association [15]. Insufficient evidence to dismiss either convergent validity or divergent validity was based on correlations between 0.30 and 0.40 [12].

Additional scales included a PtGA of disease severity and DLQI. The PtGA was a five-point scale using the same category labels as the PGA and reflects the patient's overall impression of their disease severity at a given time point. The DLQI is a validated general dermatology questionnaire, using 10 items to assess health-related quality of life $[16,17]$.

\section{Ethics approval and consent to participate}

All clinical studies were conducted in compliance with the ethical principles originating in, or derived from, the Declaration of Helsinki, and with the International Council for Harmonisation Good Clinical Practice Guidelines. All documentation was reviewed by the institutional review board and/or independent ethics committee at each of the investigational centres (Additional file 1). All patients provided written informed consent. 


\section{Results}

This analysis included data for 3641 patients with moderate to severe chronic plaque psoriasis who had participated in one of four phase III clinical studies of tofacitinib in patients with psoriasis (OPT Pivotal 1 [NCT01276639] [6], OPT Pivotal 2 [NCT01309737] [6], OPT Compare [NCT01241591] [7] and OPT Retreatment [NCT01186744] [8]). Baseline characteristics were broadly similar across all four studies. Median ages were 44-46 years (range 18-83), 68-71\% of patients were male, 81-92\% were white and median weights were 83$91 \mathrm{~kg}$ (range $36-219 \mathrm{~kg}$ ). PASI scores at baseline were similar between studies, ranging from a median of 18.4 in OPT Retreatment to 20.5 in OPT Compare. Baseline PGA scores defined $82-90 \%$ of patients as moderate and $10-17 \%$ as severe. PtGA scores were $29-35 \%$ moderate and $61-67 \%$ severe.

\section{Confirmatory Factor Analysis}

CFA is a hypothesis-confirming technique to test whether data support a hypothesised measurement model [13]. Here, the CFA model assumed that the three items (erythema, induration and scaling) are equally weighted in PGA. Results of the CFA with path coefficients associated with the three items constrained to be equal (representing the current PGA scoring algorithm), and CFA without these constraints demonstrated excellent fit, indicated by CFI values $>0.98$ and standardised path coefficients all above the threshold of 0.4 (Table 1).

Furthermore, when comparing the 'relaxed constraints' paths and the estimated common loadings, 17 out of 24 paths did not differ significantly from each other (Table 1) and those that did occurred at baseline (when there was less assurance given the limited range of the scores from a relatively homogeneous, pretreatment sample) or were not sizable enough to be meaningful at the primary time assessments. In general, these results support equal weighting of erythema, induration and scaling in the PGA scoring algorithm.

\section{Test-retest reliability}

This technique evaluated consistency of PGA measurements between screening and baseline visits, when no change in terms of disease severity was anticipated. Intraclass Correlation Coefficient values between 0.7 and 0.9 are considered to represent acceptable reliability, while values $>0.9$ are usually interpreted as representing excellent reliability $[12,13]$. The estimated Intraclass Correlation Coefficient of the PGA for the pooled data was 0.70 , thereby suggesting acceptable test-retest reliability that indicates consistency of the PGA scoring over a stable period. Intraclass Correlation Coefficient estimations calculated separately for each study were acceptable for two studies (OPT Pivotal 2, 0.79; OPT Compare, 0.70) and less so for the other two studies (OPT Pivotal 1, 0.60; OPT Retreatment, 0.65).

\section{Internal consistency reliability}

Internal consistency reliability was used to determine if scoring of erythema, induration and scaling were consistent with each other. The PGA scale rendered excellent internal consistency reliability, as indicated by a Cronbach's Coefficient Alpha $\geq 0.9$ in all calculations at the primary assessment time points (OPT Pivotal 1, 0.94; OPT Pivotal 2, 0.95; OPT Compare, 0.92; OPT Retreatment, 0.94). The Cronbach's Coefficient Alpha values observed at baseline (OPT Pivotal 1, 0.50; OPT Pivotal 2, 0.63; OPT Compare, 0.63; OPT Retreatment, 0.51 ) were smaller as a result of the pre-selection of relatively homogeneous subjects at baseline (due to the inclusion and exclusion criteria for the clinical trials) whose responses were generally within a restricted range, resulting in small pairwise correlations as expected. These findings indicated that the three item

Table 1 PGA Confirmatory Factor Analysis

\begin{tabular}{|c|c|c|c|c|c|c|}
\hline \multirow[t]{2}{*}{ Study } & \multirow{2}{*}{$\begin{array}{l}\mathrm{CFI}^{\mathrm{a}} \text { (constrained } \\
\text { paths) }\end{array}$} & \multirow{2}{*}{$\begin{array}{l}\mathrm{CF}^{\mathrm{a}} \text { (unconstrained } \\
\text { paths) }\end{array}$} & & \multicolumn{3}{|c|}{ Difference between path coefficients (constrained vs unconstrained), \% } \\
\hline & & & & Erythema & Induration & Scaling \\
\hline \multirow[t]{2}{*}{ OPT Pivotal 1} & 0.995 & 0.996 & Baseline & -18.1 & 16.7 & 4.2 \\
\hline & & & Week 16 & 0.5 & 1.9 & -1.6 \\
\hline \multirow[t]{2}{*}{ OPT Pivotal 2} & 0.983 & 0.995 & Baseline & -9.5 & $18.7^{*}$ & -5.0 \\
\hline & & & Week 16 & $-5.5^{*}$ & 5.0 & -1.8 \\
\hline \multirow[t]{2}{*}{ OPT Compare } & 0.988 & 0.997 & Baseline & $-16.8^{*}$ & $16.6^{*}$ & 0.6 \\
\hline & & & Week 12 & -3.2 & 5.5 & -3.9 \\
\hline \multirow[t]{2}{*}{ OPT Retreatment } & 0.981 & 0.994 & Baseline & $-34.3^{*}$ & $38.9^{*}$ & 0.8 \\
\hline & & & Week 24 & -6.0 & $2.8^{*}$ & 0.9 \\
\hline
\end{tabular}

$p<0.05$ vs zero

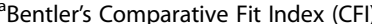

PGA Physician Global Assessment 
scores were consistent with each other and, therefore, showed concordance with each other.

\section{Clinically Important Difference}

The CID is the magnitude of change in PGA that is discernible, clinically and meaningfully, as a difference in disease severity to a patient. With PtGA as a continuous anchor, CID for the PGA score was 0.55 (95\% confidence interval: $0.546-0.563$ ), based on pooled data from all four studies, which corresponded to a one-category difference in the PtGA. Figure 2 shows a plot of the PtGA against the PGA when used as either a continuous or categorical predictor. The functional relationship between PGA and PtGA is clearly linear and positive. Results on CID from individual studies were similar (0.53 for OPT Pivotal 1 and 2, to 0.62 for OPT Retreatment), which justified the pooling of the results.

\section{Known-group validity}

Known-group validity analysis was used to assess whether PGA could discriminate between different degrees of psoriasis severity, using PASI as a measure of disease severity. A clear positive relationship between PGA and PASI scores was observed (Fig. 3a). The differences in the PGA scores between the 'clear' group (PASI score of 0 ) and the other groups (PASI score $>0$ ) were statistically significant (Table 2), and increased as psoriasis became more severe (i.e., with larger PASI scores). Differences were all greater than the CID, indicating that they were also clinically relevant.

Analysing the data for each study separately confirmed the very close relationship between PGA and PASI (Fig. 3b), which was stable and replicable across the four phase III studies (Fig. 3b). A robust monotonic functional relationship between PASI and PGA was observed, and showed that the PGA scale can separate between groups that are known to be different; in this case, a clear group (no psoriasis) and groups with psoriasis.

\section{Convergent and divergent validity}

This analysis was performed to assess whether PGA correlated sufficiently with other clinical end points used in the phase III studies; namely: PASI, PtGA and DLQI. Pearson correlation coefficients between the PGA and each of PASI, PtGA and DLQI at primary assessment time point were $>0.4$ (Table 3 ), indicating, as expected, significant and substantial correlations of PGA with these clinical end points. The correlations were not extremely large (above 0.80), indicating that PGA captures some different information compared with PASI and DLQI. In addition, it should be noted that at baseline the correlations diverged and were smaller (Pearson

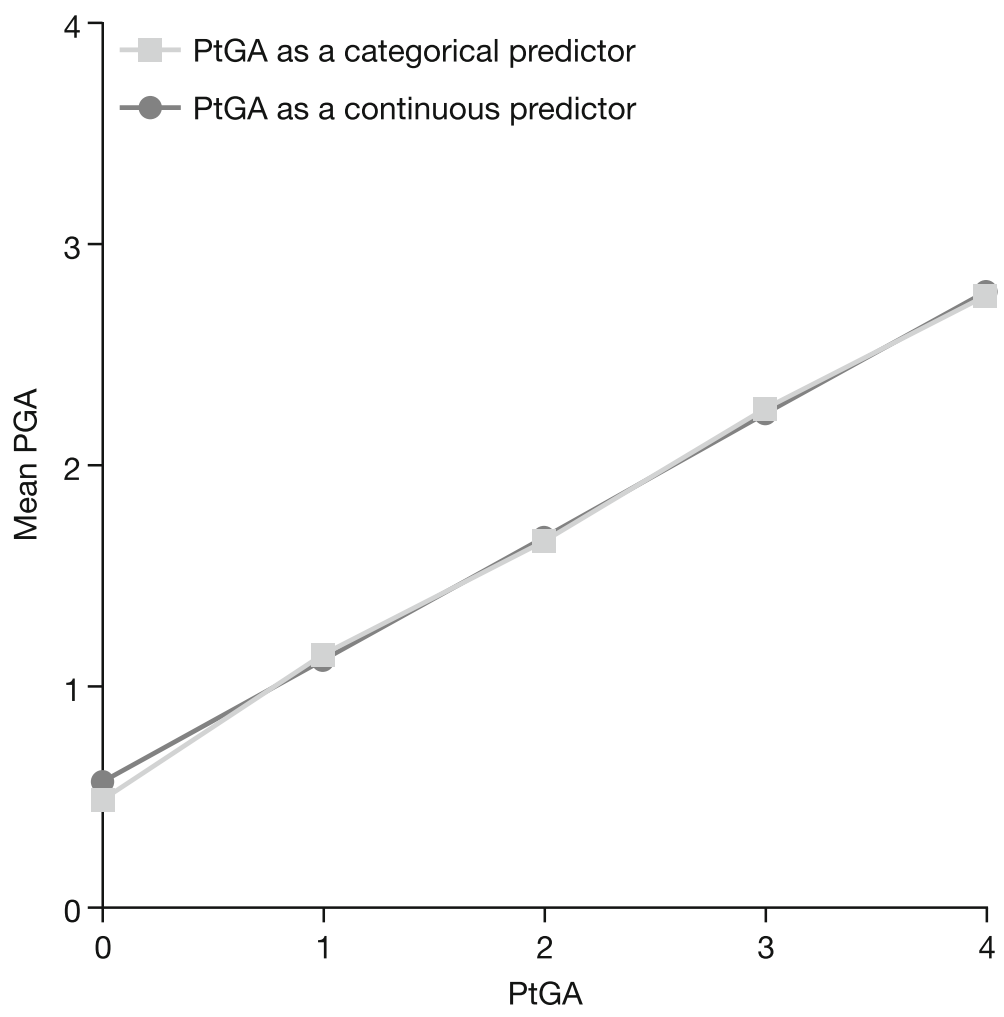

Fig. 2 Relationship between PGA score and PtGA category. PGA Physician Global Assessment, PtGA Patient Global Assessment 


\section{a}

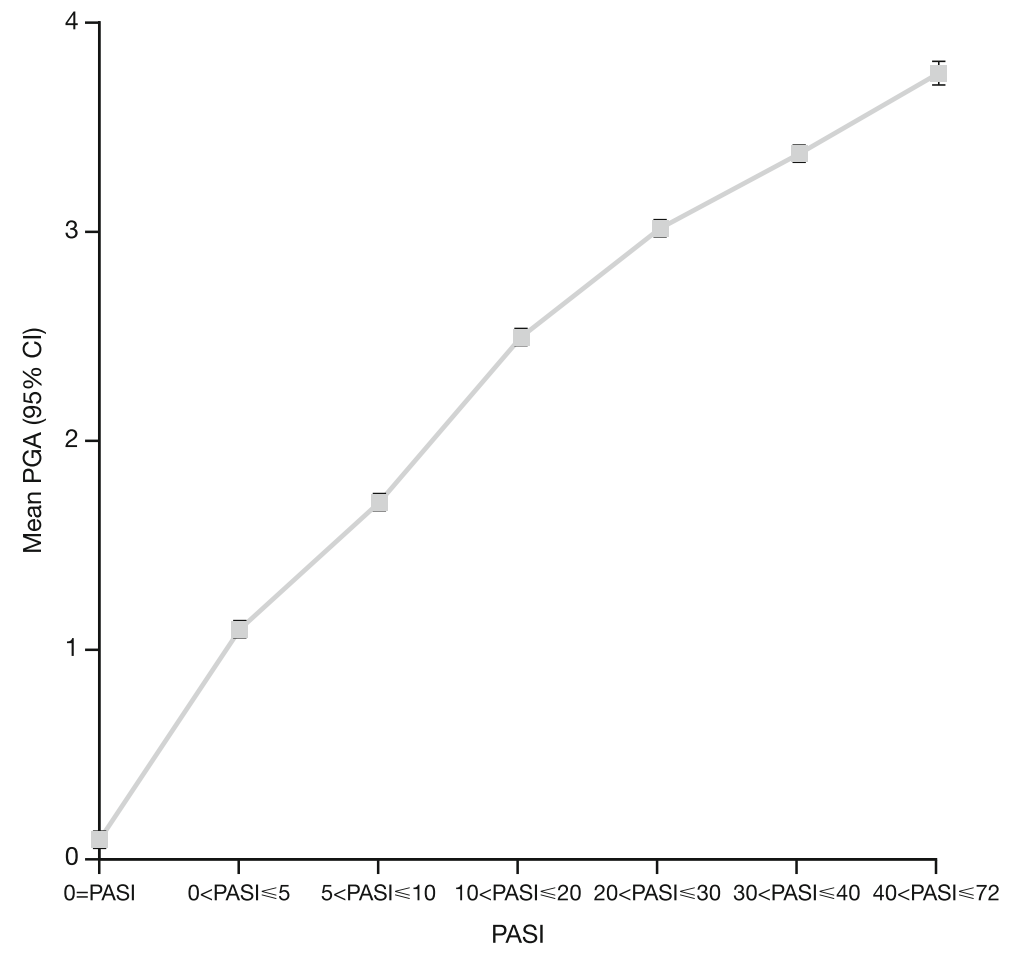

b

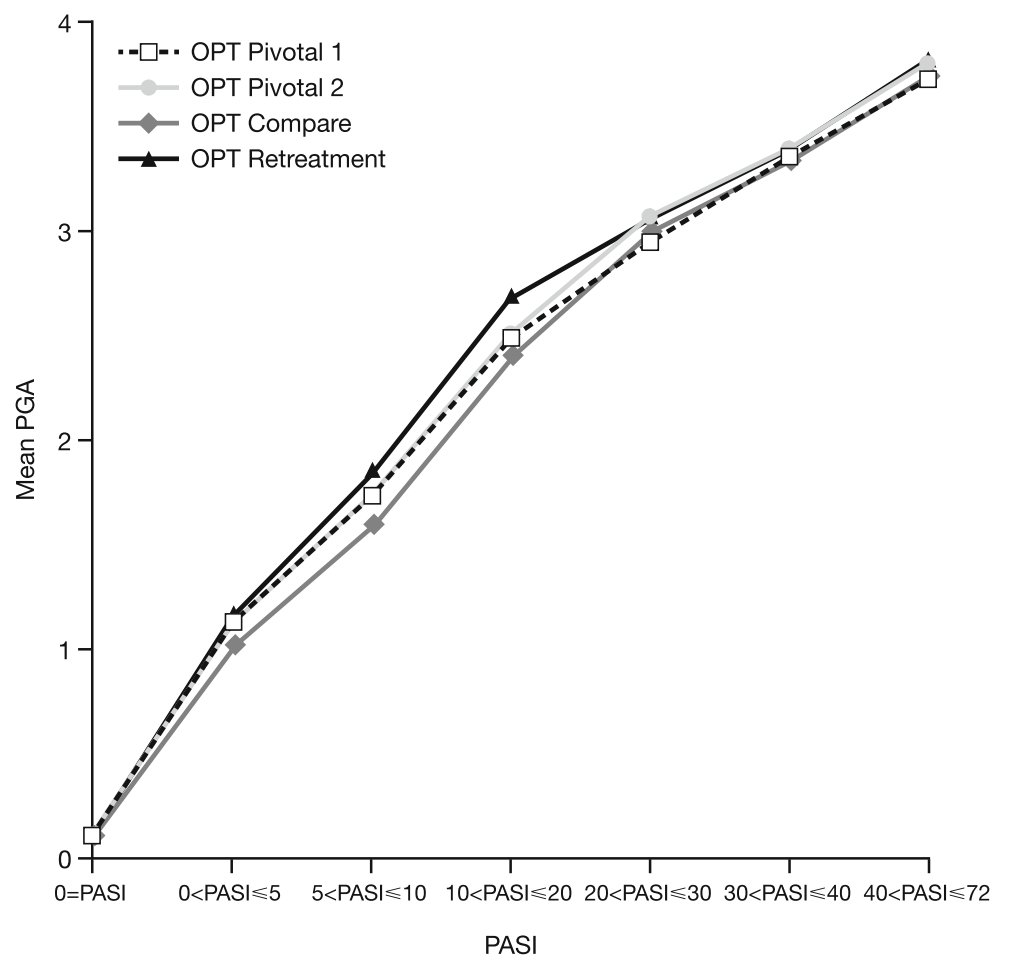

PASI

Fig. 3 Relationship between PASI score and PGA score. (a) Pooled data; (b) Individual studies. Cl confidence interval, PASI Psoriasis Area and Severity Index, PGA Physician Global Assessment 
Table 2 Known-group validity of the PGA

\begin{tabular}{|c|c|c|c|}
\hline \multirow{2}{*}{$\begin{array}{l}\text { Category definition } \\
\text { based on the } \\
\text { PASI score }\end{array}$} & \multirow{2}{*}{$\begin{array}{l}\text { Mean difference in } \\
\text { PGA scores between } \\
\text { group with PASI } \\
\text { score }=0 \text { and indicated } \\
\text { PASI score group }\end{array}$} & \multicolumn{2}{|l|}{$95 \% \mathrm{Cl}$} \\
\hline & & Lower & Higher \\
\hline $0<\mathrm{PASI} \leq 5$ & -1.01 & -1.04 & -0.98 \\
\hline $5<$ PASI $\leq 10$ & -1.61 & -1.65 & -1.58 \\
\hline $10<$ PASI $\leq 20$ & -2.41 & -2.44 & -2.37 \\
\hline $20<$ PASI $\leq 30$ & -2.92 & -2.96 & -2.89 \\
\hline $30<\mathrm{PASI} \leq 40$ & -3.27 & -3.31 & -3.23 \\
\hline $40<$ PASI $\leq 72$ & -3.67 & -3.73 & -3.61 \\
\hline
\end{tabular}

${ }^{*}$ All $p$-values $\leq 0.0001$ vs group with PASI score $=0$

PGA score was outcome and PASI score was categorical anchor, with no functional relationship imposed

Cl confidence interval, PASI Psoriasis Area and Severity Index, PGA Physician Global Assessment

correlation coefficients: PGA vs PASI, 0.28-0.39; PGA vs PtGA, 0.09-0.10; PGA vs DLQI, 0.09-0.14), as expected, stemming from the pre-selection of homogeneous subjects with a limited range of responses.

\section{Discussion}

PGA scales are commonly used as a co-primary or secondary end point to assess treatment efficacy in psoriasis clinical trials. However, multiple versions of the PGA currently exist and no standard definition has yet been established. For example, a previous study reported the validation of three measures of physicianreportedpsoriasis severity, using data from 445 patients participating in a single phase III clinical trial. The static Physician's Global Assessment (sPGA), one of the three measures, is similar to the PGA validated in the current study, in that it assesses erythema, induration and

Table 3 Pearson correlation coefficients at the primary assessment time point

\begin{tabular}{llll}
\hline Study & \multicolumn{3}{l}{$\begin{array}{l}\text { Pearson correlation coefficients } \\
\text { (Prob }>|r| \text { under HO: Rho }=0)\end{array}$} \\
& \multicolumn{2}{c}{ Number of observations } \\
\cline { 2 - 4 } & PGA vs PASI & PGA vs PtGA & PGA vs DLQI \\
\hline OPT Pivotal 1 & 0.77 & 0.70 & 0.57 \\
& $(p<0.0001)$ & $(p<0.0001)$ & $(p<0.0001)$ \\
& 798 & 790 & 789 \\
OPT Pivotal 2 & 0.79 & 0.72 & 0.53 \\
& $(p<0.0001)$ & $(p<0.0001)$ & $(p<0.0001)$ \\
& 851 & 841 & 843 \\
OPT Compare & 0.79 & 0.66 & 0.53 \\
& $(p<0.0001)$ & $(p<0.0001)$ & $(p<0.0001)$ \\
& 1027 & 1011 & 1008 \\
OPT Retreatment & 0.75 & 0.70 & 0.44 \\
& $(p<0.0001)$ & $(p<0.0001)$ & $(p<0.0001)$ \\
& 555 & 549 & 549 \\
\hline
\end{tabular}

DLQI Dermatology Life Quality Index, PASI Psoriasis Area and Severity Index, PGA Physician Global Assessment, PtGA Patient Global Assessment Italic values represent the number of observations scaling; however, while the PGA reported here uses a scale from $0=$ clear to $4=$ severe, in the sPGA described by Simpson et al., a scale from 0 to 5 is used. In both cases, each component is equally weighted and the score derived as the mean of the three components [18]. This lack of consensus partly explains why, despite their simplicity, PGA scales are not routinely used by practising clinicians. Therefore, it is important to assess the validity and reliability of each PGA scale for evaluating psoriasis disease severity.

This analysis provides further validation of the threeitem PGA tool used in the tofacitinib clinical development programme in a large patient cohort using pooled data from four phase III studies, following preliminary validation based on data from a single phase II study [4]. We confirmed the properties and scoring algorithm of the tofacitinib PGA scale. The data support equal weighting of each of the three PGA items, the three PGA items were consistent with each other and the PGA was reliable over time.

We also showed that PGA and PASI follow a similar pattern with worsening disease, PGA scores could discriminate degrees of psoriasis severity based on PASI and differences in PGA between PASI categories were clinically relevant. Together, these data provide a robust validation of the three-item PGA tool for the assessment of psoriasis.

Finally, we established the CID for this PGA scale as equal to 0.55 points. When evaluating CIDs, it is most desirable to base them on a patient-reported measure, such as the PtGA used here. Although there were differences between PGA and PtGA, they were likely due to differences in patient and physician perspectives of psoriasis severity [19]. In addition, a close to linear relationship was demonstrated between PGA and PtGA when PtGA was used as a categorical anchor, supporting the main model which imposed a linear relationship between PGA and PtGA. In sensitivity analyses using data from each of the studies separately, both for the CID and other analyses, the results for each phase III study were similar and consistent with the previously published phase II results [4], demonstrating the stability and replicability of the PGA across five studies.

This finding is also consistent with a previously published systematic review comparing PASI with various PGA scales reported in the literature, which indicated that PGA and PASI correlate well but concluded that standardisation and validation of PGA was required [20].

Although the evidence clearly shows the favourable measurement properties of this PGA scale, there are potential limitations to consider when interpreting the results of this analysis. For example, the data used were taken from patients with relatively severe disease at baseline and who were being treated within the clinical 
trial environment; therefore, caution should be used when generalising these findings to the wider psoriasis population. Similarly, as patients were recruited to the studies according to set criteria, this resulted in a relatively homogeneous population at baseline, reflected by the smaller Cronbach's Coefficient Alpha observed at baseline. Following treatment, there was a greater degree of heterogeneity in the sample and the wider variation scores allow for a more accurate assessment. However, we have not evaluated the sensitivity of PGA to beneficial treatment in this current investigation as this was found and reported elsewhere [6-8].

In addition, a strong correlation with PASI was observed, despite the fact that, unlike the PASI, the PGA does not take into account the amount of body surface area affected by psoriasis. The PGA also showed a moderately strong correlation with PtGA and DLQI. Clinical measures of disease severity such as the PGA and PASI were designed to assess clinical features of psoriasis (erythema, induration and scaling), and not necessarily to evaluate the full impact of symptoms on patients and their quality of life, thus clearly justifying the use of patient-reported as well as physician-reported outcome measures in clinical trials. In general, our findings are consistent with other evidence in the literature demonstrating a correlation between improved PGA scores and increases in patient-reported measures of health-related quality of life in patients with moderate to severe psoriasis $[21,22]$.

The PGA was designed to be a relatively more simple assessment of erythema, induration and scaling than the PASI, with its more complex scoring algorithm. However, none of the PGA's items independently and wholly represents the disease and, equally importantly, the PGA does not include quantification of the area of involvement, a critical element to be considered when assessing psoriasis severity, nor does the PGA consider the locations of individual lesions. Furthermore, the precision of PGA scores highly relies on the evaluators strictly adhering to a standard definition of the PGA. PGA scales are therefore useful to assess clinical study end points of disease severity, but are no substitute for thorough clinical assessment in routine practice. No matter how well validated the PGA is, its assessment is limited to what it is intended to measure and, therefore, its breadth must be acknowledged.

Nevertheless, the PGA remains a useful measure in clinical trials of psoriasis, particularly since it is more simple and has more intuitive severity categories, scoring and interpretation than the PASI. As this work attests, the PGA scale used in tofacitinib studies of psoriasis, with its favourable properties, and the relevant scoring algorithm (equal weighting of erythema, induration and scaling) is supported robustly by all available empirical evidence.

\section{Conclusions}

Consistent with previous findings from a phase II study, these results provide needed confirmation and reassurance for a clinician on the suitability of the three-item PGA scale as a reliable, valid measure to obtain a global assessment on the severity of plaque psoriasis in clinical studies.

\section{Additional file}

Additional file 1: List of independent ethics committees or institutional review boards. (DOCX $47 \mathrm{~kb}$ )

\section{Abbreviations}

BID: Twice daily; CFA: Confirmatory Factor Analysis; CFI: Comparative Fit Index; Cl: Confidence interval; CID: Clinically Important Difference;

DLQI: Dermatology Life Quality Index; PASI: Psoriasis Area and Severity Index; PGA: Physician Global Assessment; PtGA: Patient Global Assessment

\section{Acknowledgements}

Medical writing support, under the guidance of the authors, was provided by David Finch, PhD and Sandrine M. Dupré, PhD, at CMC Connect, a division of McCann Health Medical Communications Ltd., Macclesfield, UK, and was funded by Pfizer Inc., New York, NY, USA in accordance with Good Publication Practice (GPP3) guidelines (Ann Intern Med. 2015;163:461-4). This work was conducted in Groton, CT, USA.

\section{Authors' contributions}

KCD, AGB, JCC, LM and CM were all involved in data interpretation, and manuscript drafting, reviewing and development. AGB, JCC, and CM also substantially contributed to the design of the work, and KCD contributed to the acquisition of the data. KCD, AGB, JCC, LM and CM substantially contributed to the analysis or interpretation of data, and all authors drafted the work and/or revised it critically for important intellectual content, approved the final published version of the manuscript, and are accountable for all aspects of this work.

\section{Funding}

All studies included in this analysis were sponsored by Pfizer Inc. Both Pfizer and non-Pfizer authors have participated in the study design, data collection, data analysis and open scientific discussion of the data, its interpretation, and the development of the associated manuscript. The views and opinions expressed within the manuscript are those of all authors and do not necessarily represent those of the funding organisation.

\section{Availability of data and materials}

Upon request, and subject to certain criteria, conditions and exceptions (see https://www.pfizer.com/science/clinical-trials/trial-data-and-results for more information), Pfizer will provide access to individual de-identified participant data from Pfizer-sponsored global interventional clinical studies conducted for medicines, vaccines and medical devices (1) for indications that have been approved in the US and/or EU or (2) in programmes that have been terminated (i.e., development for all indications has been discontinued). Pfizer will also consider requests for the protocol, data dictionary and statistical analysis plan. Data may be requested from Pfizer trials 24 months after study completion. The de-identified participant data will be made available to researchers whose proposals meet the research criteria and other conditions, and for which an exception does not apply, via a secure portal. To gain access, data requestors must enter into a data access agreement with Pfizer.

\section{Ethics approval and consent to participate}

All clinical studies were conducted in compliance with the ethical principles originating in, or derived from, the Declaration of Helsinki, and with the 
International Council for Harmonisation Good Clinical Practice Guidelines. All documentation was reviewed by the institutional review board and/or independent ethics committee at each of the investigational centres (Additional file 1). All patients provided written informed consent.

\section{Consent for publication}

Not applicable.

\section{Competing interests}

KCD has served as an investigator and/or consultant for Amgen, AbbVie, Boehringer Ingelheim, Bristol-Myers Squibb, Celgene, Eli Lilly, Janssen, Stiefel, Novartis and Pfizer Inc.

AGB, JCC and CM are employees and stockholders of Pfizer Inc. LM was an employee of Pfizer Inc and held stock/stock options in Pfizer Inc at the time of the analysis.

\section{Author details}

'Department of Dermatology, University of Utah School of Medicine, Salt Lake City, UT, USA. ${ }^{2}$ Pfizer Inc, 445 Eastern Point Road, Groton, CT 06340, USA. ${ }^{3}$ Pfizer Inc, Collegeville, PA, USA.

Received: 22 September 2017 Accepted: 17 May 2019

Published online: 07 June 2019

\section{References}

1. Feldman SR, Krueger GG. Psoriasis assessment tools in clinical trials. Ann Rheum Dis. 2005;64(Suppl 2):ii65-8.

2. Spuls PI, Lecluse LL, Poulsen ML, Bos JD, Stern RS, Nijsten T. How good are clinical severity and outcome measures for psoriasis?: quantitative evaluation in a systematic review. J Invest Dermatol. 2010;130:933-43.

3. Callis Duffin K, Gottlieb AB. Outcome measures for psoriasis severity: a report from the GRAPPA 2012 annual meeting. J Rheumatol. 2013;40:1423-4.

4. Cappelleri JC, Bushmakin AG, Harness J, Mamolo C. Psychometric validation of the physician global assessment scale for assessing severity of psoriasis disease activity. Qual Life Res. 2013;22:2489-99.

5. Sloan JA, Aaronson N, Cappelleri JC, Fairclough DL, Varricchio C. Assessing the clinical significance of single items relative to summated scores. Mayo Clin Proc. 2002;77:479-87.

6. Papp KA, Menter MA, Abe M, Elewski B, Feldman SR, Gottlieb AB, et al. Tofacitinib, an oral Janus kinase inhibitor, for the treatment of chronic plaque psoriasis: results from two, randomized, placebo-controlled, phase III trials. Br J Dermatol. 2015;173:949-61.

7. Bachelez H, van de Kerkhof PC, Strohal R, Kubanov A, Valenzuela F, Lee JH, et al. Tofacitinib versus etanercept or placebo in moderate-to-severe chronic plaque psoriasis: a phase 3 randomised non-inferiority trial. Lancet. 2015;386:552-61

8. Bissonnette $R$, Iversen $L$, Sofen $H$, Griffiths CEM, Foley P, Romiti R, et al. Tofacitinib withdrawal and retreatment in moderate-to-severe chronic plaque psoriasis: a randomized controlled trial. Br J Dermatol. 2015;172: 1395-406.

9. Brown TA. Confirmatory factor analysis for applied research. New York, NY: The Guilford Press; 2006.

10. Hatcher L. A step-by-step approach to using the SAS ${ }^{\circledast}$ system for factor analysis and structural equation modeling. Cary, NC: SAS Institute Inc.; 1994.

11. Kline R. Principles and practice of structural equation modeling. 2 nd ed. New York, NY: The Guilford Press; 2005.

12. Fayers FM, Machin D. Quality of life: the assessment, analysis, and interpretation of patient-reported outcomes. 2nd ed. Chichester: John Wiley and Sons Ltd; 2007.

13. Cappelleri JC, Zou KH, Bushmakin AG, Alvir JMJ, Alemayehu D, Symonds T. Patient-reported outcomes: measurement, implementation and interpretation. Boca Raton, FL: Chapman \& Hall/CRC; 2013.

14. Stevens J. Applied Multivariate statistics for the social sciences. Hillsdale, NJ: Lawrence Erlbaum; 2002.

15. Cohen J. Statistical power analysis for the behavioral sciences. 2nd ed Hillsdale, NJ: Lawrence Erlbaum; 1988.

16. Finlay AY, Khan GK. Dermatology Life Quality Index (DLQI)—a simple practical measure for routine clinical use. Clin Exp Dermatol. 1994;19:210-6.

17. Mazzotti E, Picardi A, Sampogna F, Sera F, Pasquini P, Abeni D. Sensitivity of the Dermatology Life Quality Index to clinical change in patients with psoriasis. Br J Dermatol. 2003;149:318-22.
18. Simpson MJ, Chow C, Morgenstern H, Luger TA, Ellis CN. Comparison of three methods for measuring psoriasis severity in clinical studies (part 2 of 2): use of quality of life to assess construct validity of the Lattice System Physician's Global Assessment, Psoriasis Area and Severity Index and Static Physician's Global Assessment. J Eur Acad Dermatol Venereol. 2015;29:1415-20.

19. Paul C, Bushmakin AG, Cappelleri JC, Mallbris L, Mamolo C. Do patients and physicians agree in their assessment of the severity of psoriasis? Insights from tofacitinib phase 3 clinical trials. J Dermatolog Clin Res. 2015;3:1048.

20. Robinson A, Kardos M, Kimball AB. Physician Global Assessment (PGA) and Psoriasis Area and Severity Index (PASI): why do both? A systematic analysis of randomized controlled trials of biologic agents for moderate to severe plaque psoriasis. J Am Acad Dermatol. 2012;66:369-75

21. Takeshita J, Callis Duffin K, Shin DB, Krueger GG, Robertson AD, Troxe $A B$, et al. Patient-reported outcomes for psoriasis patients with clear versus almost clear skin in the clinical setting. J Am Acad Dermatol. 2014;71:633-41.

22. Viswanathan HN, Chau D, Milmont CE, Yang W, Erondu N, Revicki DA, et al. Total skin clearance results in improvements in health-related quality of life and reduced symptom severity among patients with moderate to severe psoriasis. J Dermatolog Treat. 2015;26:235-9.

\section{Publisher's Note}

Springer Nature remains neutral with regard to jurisdictional claims in published maps and institutional affiliations.
Ready to submit your research? Choose BMC and benefit from:

- fast, convenient online submission

- thorough peer review by experienced researchers in your field

- rapid publication on acceptance

- support for research data, including large and complex data types

- gold Open Access which fosters wider collaboration and increased citations

- maximum visibility for your research: over $100 \mathrm{M}$ website views per year

At BMC, research is always in progress.

Learn more biomedcentral.com/submissions 